Derecho comercial 


\title{
Las sociedades anónimas deportivas como sucedáneas de las obsolescentes asociaciones civiles en el ámbito del derecho deportivo
}

\author{
José Antonio Arostegui Hirano \\ y Jorge Armando Díaz Montalvo
}

\section{Problemas estructurales: la formación de las asociaciones civiles y su estancamiento institucional}

Existen en el fútbol mundial distintos parámetros evaluadores y rankings que buscan distinguir a los clubes deportivos a partir de su competitividad y buen manejo, con lo que logran evidenciar las experiencias más exitosas, para que se emulen sus criterios de gestión y continuo cambio.

En el polémico ranking ${ }^{1}$ de la International Federation of Football History and Statistics (IFFHS) se encuentran solo dos equipos del Perú en el grupo de los cien primeros: San Martín y Cienciano. Si bien estas ubicaciones podrían resultar alentadoras, entendemos que dicha clasificación se basa, sobre todo, en el acceso a competencias internacionales, además de ser un consolidado histórico de participaciones y resultados.

\footnotetext{
Ranking IFFHS, 1st February 2008 - 30th January 2009.
} 
Al parecer, este criterio tuvo más peso en la calificación que se le hiciera a Cienciano, que venía de conseguir años atrás la Copa Sudamericana y la Recopa, competiciones que hicieron que su puntaje consolidado lo mantuviera en una posición más que aceptable. En el caso de la San Martín se incluyó una evaluación sobre su gestión institucional, en tanto se trataba de una institución "joven", la que se apreciaba sólida y que iba de la mano de buenos resultados.

Lo expuesto nos lleva a preguntarnos qué viene sucediendo con el resto de equipos del campeonato local; con aquellos "clubes grandes" que, si bien cabe reconocer su aporte histórico al fútbol nacional, parecen no alcanzar resultados de reconocimiento internacional que los erijan en el ranking estadístico con indicadores reales y tangibles y como sólidas instituciones futbolísticas.

Si realizáramos un ranking mundial donde se calificaran resultados, labor institucional, pero, sobre todo, nivel de gestión y potencial financiero, ningún club deportivo del Perú podría, siquiera, ser tomado en cuenta.

En el fútbol mundial hay cosas que permanecen en el tiempo; elementos subjetivos que le dan a un club la lealtad de sus seguidores, la identificación plena de sus jugadores y los anhelos dirigenciales de verlo grande (lo que es posible a partir de una gestión seria, de largo plazo, para construir las bases de un club competitivo y ganador). Sin embargo, es necesario decir que el fútbol ha cambiado y que en el Perú se requiere una reforma institucional que les permita a los clubes alcanzar protagonismo en las competencias internacionales.

Durante muchos años nuestras instituciones han sido manejadas por círculos que se mantienen año tras año e impiden el inicio de cambios serios y sostenibles. El manejo de los clubes no ha variado mucho en bastante tiempo (salvo la inclusión de mayor publicidad), y es necesario reconocer que la plena responsabilidad de esta situación recae tanto en dirigentes como en jugadores e hinchas del club.

Estas taras se mantienen en la mayoría de instituciones y clubes deportivos de fútbol. Salir del estancamiento generalizado exige una pronta adaptación a los requerimientos que el fútbol como deporte internacional reclama. 
La empresa Apoyo Consultoría ${ }^{2}$ ha explorado, con base en los ejes de la problemática del fútbol peruano, sus principales deficiencias desde sus instituciones, demostrando cuán ineficientes han llegado a ser las asociaciones civiles como entes deportivos, las que terminan favoreciendo solo a sus asociados y no al club como institución.

En el caso de las asociaciones deportivas, graves deficiencias las muestran como instituciones con "una directiva sin responsabilidades futuras sobre la gestión presente (...) [inmersa en] una espiral de endeudamiento (...) un sentido de propiedad difuso con responsabilidad del club por los dirigentes (...) [y] un cumplimiento de las metas deportivas sin importar el costo".

A esta forma de organización societaria se la ha acusado de obedecer criterios que se agotan en el corto plazo. Los plazos de acción de una directiva y las decisiones que ésta toma a nombre del club se agotan en el marco de responsabilidad dentro de su periodo de ejercicio. Es necesario que se desarrolle, además, un horizonte de metas a largo plazo, a partir del cual se determinen los lineamientos generales de gestión institucional propios del club, para que puedan ser desarrollados consecutivamente por cada directiva elegida.

También se requiere que los clubes accedan a nuevo financiamiento, como en el caso - más adelante volveremos a él- de México, a través de socios inversores que inyecten nuevo capital a los clubes en quiebra, o de Argentina, mediante la figura del gerenciamiento, que asume una visión más planificada de la gestión.

Además de que en él no existe una relación sólida y responsable entre dirigente (gestión), fuente (inversión) y club (rentabilidad), en el fútbol peruano es una práctica común que las asociaciones civiles recurran a los fondos del club para afrontar de modo facilista e inmediato problemas de coyuntura que implican gastos que desestabilizan su presupuesto a lo largo del año.

APOYO CONSULTORÍA. Desarrollo e implementación de una estrategia para la mejora de la competitividad e incremento de la rentabilidad en el fútbol peruano. Lima, junio del 2004, p. 12. 


\section{Problemas económicos: crisis de financiamiento e ingresos}

Uno de los primeros pasos para lograr un cambio en las asociaciones civiles se relaciona con el financiamiento. La experiencia de la posible desafiliación de la Federación de Fútbol, con el consiguiente cierre de nuestra participación en cualquier torneo internacional, clausuraba la posibilidad de acceder a premios y a anunciantes, haciendo aun menos atractivo el torneo local. Tal circunstancia podía afectar, por un lado, a un gran número de jugadores, quienes ya no podrían laborar en sus clubes ante la falta de pago, y, por otro, a muchas instituciones, que se verían impedidas de mantener sus equipos y sus instalaciones ante la ausencia de sponsors y premios provenientes de la clasificación a torneos internacionales. La solidez económica de la mayoría de clubes deportivos peruanos se sustenta en estos conceptos. Por otra parte, no brinda seguridad el que una sola persona natural o jurídica garantice mes a mes que el club cuente con lo necesario para actividades mínimas como entrenamientos y compra de implementos y servicios (caso del Sport Boys del Callao, ya descendido).

Sin embargo, si se considerase que una de las salidas de la falta de ingresos puede provenir de las taquillas, ello no es así. Las taquillas en el fútbol profesional han descendido año tras año, y no porque el aficionado haya perdido interés en seguir a su equipo, sino que no tenemos equipos competitivos debido a problemas en sus presupuestos que les impiden contratar jugadores de primera para brindar un espectáculo que incentive la asistencia de un mayor número de espectadores.

La venta de jugadores es una importante fuente de financiamiento que, aun cuando es temporal, aporta al club un ingreso significativo. No obstante, son pocos los clubes peruanos que desde sus divisiones inferiores produzcan talentos que resulten "enajenables" al extranjero.

\section{Problemas deportivos: pérdida de categoría y falta de competi- tividad}

En el fútbol peruano hay clubes en cuyas instituciones se agravan las deficiencias descritas. El de los equipos que ascienden a primera división profesional es un caso emblemático: la mayoría solo se mantiene en esta categoría un año.

En los clubes "chicos" y "medianos" la situación resulta bastante crítica. Las carencias en infraestructura, trabajo físico con profesionales, medi- 
camentos y servicios de traslado y hospedaje no aseguran un mínimo de identificación con la institución y brindan un clima laboral inadecuado que no se condice en absoluto con condiciones de trabajo elementales que el empleador debe proveer a sus dependientes, en armonía con el derecho laboral, y que constituyen un primer pilar de la competitividad.

Tampoco cerrar la puerta a refuerzos de experiencia es algo que permita que el nivel de estos equipos se equilibre en relación con el de los "grandes". La transformación de las asociaciones civiles en un sistema societario de aportes regulares podría constituir una salida eficaz para los llamados clubes "chicos".

\section{Análisis de las nuevas formas de organización societaria. La experiencia internacional}

En Argentina, las observaciones que hemos hecho sobre los clubes deportivos como asociaciones civiles son compartidas en muchas críticas por los especialistas del derecho deportivo. Hay quienes incluso las han denominado como instituciones legales idóneas "para el proceso de fundación de clubes deportivos en la primera mitad del siglo XX". ${ }^{3}$

$Y$ es que en la mayoría de sociedades en donde el futbol y sus clubes han constituido un pilar importante de su sociedad se reconoce una mutación en la configuración societaria de las instituciones, situación que el argentino Ricardo Frega Navía describe como un cambio paulatino "[que] fue mutando paulatinamente desde los 70 a la fecha, [y fue posible] sobre todo con la aparición de fuertes sumas de dinero en concepto de transmisiones televisivas, traspasos internacionales de futbolistas por cifras multimillonarias, explotación del merchandising, derechos de publicidad, etc."

Algunos de nuestros clubes cuentan con las fuentes de financiamiento citadas - la mayoría solo reciben algunas para su sustento-, no obs-

FREGA NAVÍA, Ricardo. “Hacia un nuevo modelo económico en el deporte profesional: asociaciones civiles deportivas, sociedades anónimas deportivas y gerenciamiento". Cuadernos de Derecho Deportivo 1. Buenos Aires: ADHOC/ Villela Editor, 2001, p. 16. 
tante lo cual seguimos con las mismas deficiencias, lo que nos impide destacar internacionalmente. De allí que surjan nuevas preguntas respecto a la fórmula legal asociación civil: ¿qué se le permite hacer al club con sus fuentes de financiamiento bajo esta figura legal? ¿Puede ser eficiente en el manejo de sus recursos?

Ante ello, queda decir que

... los clubes, incluso los más pequeños, manejan presupuestos superiores a la mayoría de las Pymes (...). Por tanto, no es razonable dejar su dirección y explotación económica a una estructura organizacional amateur y carente de toda finalidad lucrativa, como asimismo a directivos que, más allá de su tesón y buena voluntad [porque desde luego los hay] no tienen tiempo, y en algunos casos, la idoneidad de gestión, para capitanear los destinos de los clubes. Todo ello sin contar aquellos casos en que ciertos dirigentes obran pensando en hacer negocios propios a costa del club, cayendo en actos que rozan lo delictivo. ${ }^{4}$

En nuestro medio, pequeños grupos vienen manejando los clubes, administrando sus recursos y desarrollando sus fines al libre albedrío de unos cuantos dirigentes, lo que obstaculiza la consideración del club como una institución grande que merece y debe ser dirigida y gerenciada por profesionales en la toma de decisiones financieras y administrativas. De lo que se trata es de pasar de una idea barrial de club a algo más corporativo y gerenciable.

\section{Gerenciamiento o asociaciones civiles deportivas}

El año 2000 se trató de salvar a las entidades deportivas argentinas - que afrontaban una seria crisis económica - con el denominado Plan de Recuperación mediante Inversiones Privadas en el Futbol Profesional. Esta reglamentación entró en vigencia para los clubes deportivos de fútbol en marzo de ese año. La norma, en el punto uno de su segundo párrafo, definía el gerenciamiento:

4 Op. cit. 
... consiste en estructurar un régimen articulado de relaciones voluntarias entre clubes profesionales que se encuentran en aquella situación [de crisis] con otras personas jurídicas determinadas, con capacidad de aportar los recursos necesarios para llevar adelante un proyecto común de viabilidad, centrado en la gestión de la actividad del futbol profesional del club con el que se vincula, dentro de un marco de responsabilidad jurídico económica que garantice la estabilidad patrimonial y el normal desarrollo de la vida de las instituciones... ${ }^{5}$

Es decir, un agente ajeno al club sería el que lo gerenciara, aportando una fuerte suma de dinero. Barbieri desarrolla la figura estableciéndola como "una relación contractual entre el club gerenciado y la persona jurídica gerenciante". ${ }^{6}$ Este tipo de contrato se encontraría regido con una "libertad atenuada, es decir que se permite un amplio margen de contratación (...) pero con determinados límites", 7 los que pasamos a desarrollar en las siguientes líneas.

5.1. (...) La persona jurídica "gerenciante" debe tener como objeto central el desarrollo de las actividades pactadas en el contrato, constituir domicilio en la misma jurisdicción del club y contar con la solvencia moral, patrimonial y económica que permita llevar a buen término el plan de viabilidad...

Por un lado, resulta esencial que, por seguridad, el club y el "gerenciante" tengan su domicilio en la misma jurisdicción. Desde luego, deberá existir una comisión dentro de la Federación Peruana de Fútbol, que evalúe, como garante, el nivel de factibilidad que el plan de viabilidad tenga en cifras y manejos reales. Emularía lo que en la experiencia argentina se ha llamado "Comité de Evaluación de la Factibilidad".

5 BARBIERI, Pablo. "Plan de recuperación mediante inversiones privadas en el fútbol profesional", publicado el 8 de marzo del 2000. Citado en BARBIERI, Pablo. Fútbol y derecho. Buenos Aires: Editorial Universidad, 2001, p. 230.

6 Ibíd., p. 231.

7 Op. cit. 
Por otro lado, será importante el diseño y negociación del contrato, pues la junta de asociados deberá sentarse en igualdad de condiciones frente a la otra parte, el "gerenciante", quien posee y llega al club con un poder económico suficiente como para imponer condiciones. Es a su vez esencial que todos los asociados cuenten con, y den, una opinión informada al respecto, para que no se generen expectativas sobre situaciones irreales; toda decisión debe involucrar un conocimiento pleno de la información (es lo que se denomina "proceso de sinceramiento de las partes"). El proceso de negociación marcará los alcances de lo que el "gerenciante" quiere hacer en específico con el club, los alcances de su responsabilidad por ello, lo que la junta de asociados espera que aquel concrete con su gestión, así como los medios para intimarle el cumplimiento. $Y$ es que se constituyen como límites del contrato: "el plazo y las condiciones de ampliación o extinción, los bienes y derechos implicados en la relación contractual, las contraprestaciones económicas, las garantías de cumplimiento de las condiciones, los mecanismos de control dispuestos a favor del club y, por último, la protección del patrimonio del club y de su vida institucional frente a cualquier circunstancia, dando prioridad al respeto de los derechos individuales de sus socios". ${ }^{8}$

5.2. (...) Cada persona jurídica "gerenciante" podrá trabar una única relación contractual de esta naturaleza, es decir con un solo club...

Detrás de esta disposición de la norma argentina de salvataje existe un fundamento importante: evitar un futuro conflicto de intereses.

5.3. (...) Debe establecerse de qué modo se cancelarán los pasivos de la entidad afiliada...

Y es que se debe considerar una sólida transparencia respecto a la viabilidad financiera que mencionáramos líneas arriba, para que "[el

8 Op. cit. 
gerenciamiento] no se convierta en una mera actividad especulativa", ${ }^{\prime}$ lo cual, a partir del actual momento económico y/o financiero que atravesamos, resultaría catastrófico.

Frega Navía considera que el gerenciamiento es una posición intermedia o híbrida, denominada "Asociaciones Civiles Deportivas", ${ }^{10}$ entre dos salidas posibles: "un primer modelo [es decir, el de las asociaciones civiles], que reduce las expectativas respecto del nivel de los torneos locales", y "un segundo modelo [como el de las sociedades anónimas deportivas, denominadas "SAD"], que da cabida a los capitales externos [debiendo establecerse] los controles necesarios de gestión"; ;1 que ofrece algunos inconvenientes en la denominación, pues no se agota en el gerenciamiento propiamente dicho, sino que va, como un negocio jurídico, mucho más allá.

Como fenómeno, el gerenciamiento se dio en uno de los clubes más influyentes de México, el Deportivo Toluca F.C., que presenta una historia asombrosa en relación con la planificación y los resultados del club una vez que asumiera el cargo de presidente - estableciendo un modelo base de gerenciamiento - el señor Rafael Lebrija Guiot, quien a partir de 1997 dio mucha competitividad al equipo, permitiéndole ganar los torneos locales y participar con protagonismo en competencias internacionales. Tras once años de gestión, el Toluca es un club sólido y de una visión clara hacia el futuro. Esta experiencia abrió paso a la incorporación, en la mayoría de clubes, de socios estratégicos - personas naturales o jurídicas-, quienes ingresaban aportando fuertes sumas de dinero, formando fideicomisos que gestionarían y administrarían.

\section{Las sociedades anónimas deportivas (SAD)}

La innovación en esta forma de constitución societaria deportiva es la sociedad española. La ley 10/1990 disponía, en su preámbulo: ${ }^{12}$

9 FREGA NAVÍA, Ricardo. “Hacia un nuevo modelo económico en el deporte profesional...". Op. cit., p. 22.

${ }^{10} \quad$ Ibíd., p. 20.

11 Op. cit.

12 Real Decreto 10/1990, revisado en <http://noticias.juridicas.com/base_datos/ Admin/110-1990.html> 
... En un primer nivel, la Ley propone un nuevo modelo de asociacionismo deportivo que persigue, por un lado, favorecer el asociacionismo deportivo de base, y por otro, establecer un modelo de responsabilidad jurídica y económica para los clubes que desarrollan actividades de carácter profesional. Lo primero se pretende lograr mediante la creación de clubes deportivos elementales, de constitución simplificada. Lo segundo, mediante la conversión de los clubes profesionales en Sociedades Anónimas Deportivas, o la creación de tales sociedades para los equipos profesionales de la modalidad deportiva que corresponda, nueva forma jurídica que, inspirada en el régimen general de las sociedades anónimas, incorpora determinadas especificidades para adaptarse al mundo del deporte...

Esta norma fue derogada por el Real Decreto 1251/1999, del 16 de julio el mismo año, que tenía

... por finalidad básica la de aproximar el régimen jurídico de las sociedades anónimas deportivas al del resto de entidades que adoptan esta forma societaria, permitiendo una futura cotización de sus acciones en las Bolsas de Valores y, simultáneamente, establecer un sistema de control administrativo sobre el accionariado y la contabilidad de estas sociedades, con el fin de velar por la pureza de la competición y proteger los intereses públicos y de los potenciales inversores... ${ }^{13}$

Es decir, se abría la posibilidad de que los clubes actuaran como sociedades anónimas, poniendo en la bolsa de valores sus acciones y cotizándolas como cualquier empresa.

La norma establece la obligación de que se transformen en SAD todos aquellos clubes que deseen participar en torneos oficiales de la liga. ${ }^{14} \mathrm{La}$

13 Real Decreto 1251/1999, revisado en <http://noticias.juridicas.com/base_datos/ Privado/rd1251-1999.html\#da8>

14 Salvo aquellos equipos que tuvieron, en los últimos cuatro ejercicios anteriores a la ley, un patrimonio neto positivo. Real Madrid, Barcelona, Osasuna y Atlético Bilbao se exceptuaron en ese entonces. 
disposición adicional quinta obliga, además, a los clubes que asciendan a la primera división del fútbol español, a adoptar el modelo. La ley asimismo define y complementa la reglamentación que se les diera a dichas sociedades a través del Real Decreto 1084, en 1991.

En Argentina también se presentaron dos proyectos de ley con el modelo español: uno en 1996 y otro que fue posteriormente un anteproyecto del Ministerio de Justicia que establecía las SAD en forma facultativa y solo en los casos de instituciones deportivas profesionales.

Pero veamos las posiciones que se tienen respecto a las SAD como alternativa viable para su aplicación a los clubes profesionales de fútbol.

Por un lado, hay quienes han afirmado que las SAD contribuirían a "incrementar la recaudación tributaria del Estado"15 y que "las normas contables por las que se rigen las asociaciones civiles no permiten el registro claro y real de determinados valores patrimoniales intangibles (...) la contabilidad exigida a las sociedades anónimas resulta más realista". ${ }^{16}$

A propósito de lo anterior, debe decirse que el modelo de sociedades anónimas deportivas haría que muchos de los clubes ya no estuvieran exentos de ciertos impuestos que, como asociaciones, no tributan. Sin embargo, valores millonarios como el merchandising, la sponsorización, la transmisión televisiva y los contratos podrían manejarse contablemente de un modo más transparente si se instaura una contabilidad propia de la sociedad anónima.

También están los que acotan que "En la actualidad, los clubes de fútbol constituyen una verdadera unidad empresarial y como tal deben tener un marco regulador dentro del régimen de sociedades comerciales". ${ }^{17} \mathrm{Y}$ es que - como han manifestado muchos dirigentes que asumie-

Ver FREGA NAVÍA, Ricardo. “Hacia un nuevo modelo económico en el deporte profesional...". Op. cit., p. 17.

15 Ibíd., p. 19.

16 BARBIERI, Pablo. Fútbol y derecho. Buenos Aires: Editorial Universidad, 2001, pp. 86 y 87.

17 Op. cit. 
ron este modelo- resulta incongruente que una actividad que de un tiempo a esta parte mueve tanto capital se mantenga en regímenes sin fines de lucro. El carácter subjetivo de las instituciones, desde luego, permanece, pero es cierto que el fútbol ya ha pasado - desde hace buen tiempo- a ser un deporte comercial.

Por otro lado, en la posición contraria están los argumentos según los cuales asumir las SAD significaría "que las asociaciones deportivas nacionales perderían buena parte de su poder [en el caso del Perú, la FPF], ya que estas asociaciones civiles sin fines de lucro no podrán ser el órgano rector en conjunto de las SAD".$^{18}$

En nuestra realidad no resulta desventajosa la pérdida de dicho poder por parte de la Federación Peruana de Fútbol. Dejarle únicamente el control de los aspectos técnico-deportivos - como en Españapodría significar una solución a los problemas dirigenciales y de gestión que se presentan en ella.

Empero, sí es importante considerar que "las anomalías que se detectan en la administración de las asociaciones civiles tienen su correlato en otras anormalidades que se registran en algunas sociedades anónimas", por el nivel de factibilidad y viabilidad financiera de la empresa "gerenciante". Preocupa su licitud y transparencia, pues existen ejemplos de "accionistas escondidos bajo sociedades inscritas en países exóticos (...), directores insolventes que no garantizan su gestión, grupos económicos que se apropian de sociedades con objetivos a veces oscuros, etc.". ${ }^{19}$

También hay que tener en cuenta los intereses económicos que el "gerenciante" mantiene para recuperar su inversión, y cómo lograr que armonicen y no se antepongan a los objetivos y planes de "los asociados, socios, hinchas y simpatizantes de los distintos clubes" ${ }^{20}$

En opinión de muchos especialistas argentinos, entre ellos Pablo Barbieri, ${ }^{21}$ la situación caótica de los clubes es consecuencia de elemen-

18 FREGA NAVÍA, Ricardo. “Hacia un nuevo modelo económico en el deporte profesional...". Op. cit., p. 19.

19 BARBIERI, Pablo. Fútbol y derecho. Op. cit., p. 88.

20 Op. cit.

${ }^{21} \quad$ Ibíd., pp. 90-92. 
tos como "un funcionamiento dentro de una economía en franco proceso recesivo y con escasez de recursos (...) una administración irresponsable por parte de dirigentes (...) distribución inequitativa de los ingresos televisivos [aquí resalta el tema de los clubes en ascenso] (...) la disminución de público concurrente por la violencia imperante (...)"; los cuales, sin embargo, no "son atribuibles directamente a la estructura jurídica de las asociaciones civiles como organización de los clubes de fútbol".

Si bien existen elementos que nos asemejan con la situación argentina, nuestra realidad transita por algo distinto. Uno de los problemas esenciales en nuestro medio es la labor dirigencial y la forma en que se vienen tomando las decisiones en la mayoría de clubes respecto a sus planes a largo plazo y sus objetivos; estas consideraciones sí requieren una reconfiguración en el sentido mismo de la institución deportiva como figura legal. 


\section{Bibliografía}

APOYO CONSULTORÍA. Desarrollo e implementación de una estrategia para la mejora de la competitividad e incremento de la rentabilidad en el fútbol peruano. Lima, junio del 2004.

BARBIERI, Pablo. Fútbol y derecho. Buenos Aires: Editorial Universidad, 2001.

BARBIERI, Pablo. "Plan de recuperación mediante inversiones privadas en el fútbol profesional", publicado el 8 de marzo del 2000. Citado en BARBIERI, Pablo. Fútbol y derecho. Buenos Aires: Editorial Universidad, 2001.

FREGA NAVÍA, Ricardo. "Hacia un nuevo modelo económico en el deporte profesional: asociaciones civiles deportivas, sociedades anónimas deportivas y gerenciamiento". Cuadernos de Derecho Deportivo 1. Buenos Aires: ADHOC/ Villela Editor, 2001. 